

\title{
Gaussian beams for quasi-optical-systems: Modeling dichroic surfaces near a zero of transmission or reflection
}

Alexandre Chabory, Kevin Elis, Jérôme Sokoloff, Sylvain Bolioli

\section{To cite this version:}

Alexandre Chabory, Kevin Elis, Jérôme Sokoloff, Sylvain Bolioli. Gaussian beams for quasi-opticalsystems: Modeling dichroic surfaces near a zero of transmission or reflection. EMTS 2016, URSI International Symposium on Electromagnetic Theory, Aug 2016, Espoo, Finland. pp.Electronic ISBN: 9781-5090-2502-2 Print on Demand(PoD) ISBN: 978-1-5090-2503-9, 10.1109/URSI-EMTS.2016.7571310 . hal-01372300

\section{HAL Id: hal-01372300 \\ https://hal-enac.archives-ouvertes.fr/hal-01372300}

Submitted on 24 Oct 2016

HAL is a multi-disciplinary open access archive for the deposit and dissemination of scientific research documents, whether they are published or not. The documents may come from teaching and research institutions in France or abroad, or from public or private research centers.
L'archive ouverte pluridisciplinaire HAL, est destinée au dépôt et à la diffusion de documents scientifiques de niveau recherche, publiés ou non, émanant des établissements d'enseignement et de recherche français ou étrangers, des laboratoires publics ou privés. 


\title{
Gaussian Beams for Quasi-Optical-Systems: Modeling Dichroic Surfaces near a Zero of Transmission or Reflection
}

\author{
Alexandre Chabory ${ }^{1}$, Kevin Elis ${ }^{2}$, Jérôme Sokoloff ${ }^{3}$, Sylvain Bolioli ${ }^{4}$ \\ ${ }^{1}$ ENAC, TELECOM-EMA, F31055 Toulouse, France \\ Université de Toulouse, F-31400 Toulouse, France \\ 2 Antennas Group, CNES, 9 av. Edouard Belin, 31055 Toulouse, France \\ ${ }^{3}$ LAPLACE, Université de Toulouse, UPS, F-31062 Toulouse, France \\ ${ }^{4}$ ONERA, DEMR, 2 av. Edouard Belin, F-31055 Toulouse, France
}

\begin{abstract}
Gaussian-beam based methods are well-known for modeling quasi-optical systems constituted by mirrors, lenses, and horn antennas. In this article, a solution is proposed to include dichroic surfaces while maintaining the Gaussian-beam formulation. Special attention is devoted to the case where the dichroic surface response presents a zero near the angle of incidence. Numerical experiments are led on a dielectric slab to show the efficiency of the method.
\end{abstract}

\section{Introduction}

Millimeter-wave devices can include quasi-optical systems constituted by various elements, e.g. mirrors, lenses, dichroic surfaces, horn antennas. The size and the number of elements makes beam-based methods appropriate to tackle the modeling of theses systems [1]. For example, in [2] the incident field is expanded in terms of Gaussian beams, and afterward the beams are tracked through mirrors and lenses. To account for dichroic surfaces, a specific solution has been proposed in $[3,4]$.

In this article, we propose a Gaussian-beam based method for modeling quasioptical systems constituted by mirrors, lenses, dichroic surfaces, and horn antennas. More specifically, the method to include dichroic surfaces in [3] is extended to the case where either the reflection or transmission coefficient has a zero near the angle of incidence.

In Section 2, the expansion and shooting are briefly presented. In Section 3, the method to deal with the transmission and reflection through a dichroic surface is detailed in 2D. In Section 4, numerical experiments are led to test the validity of the method. 


\section{Gaussian Beam Shooting Algorithm}

\subsection{Expansion}

The expansion of a field in terms of Gaussian beams can be realized using Gabor frames/bases, which consist in representing the field on a phase-space discrete lattice [5]. To reduce the number of beams, physics-based expansions can also be used [6]. In this case, a set of beams, which parameters are chosen accordingly with the configuration, are matched with the initial field on a matching interface.

\subsection{Shooting algorithm}

To deal with the interaction of Gaussians beams with dielectric or metallic interfaces, a phase-matching approach can be used [7]. This consists in assuming that one incident Gaussian beam yields one transmitted and one reflected Gaussian beams. This leads to Gaussian-beam shooting algorithm where each beam generated by the expansion of the incident field is tracked through mirrors and lenses [2]. The stopping criterion of the tracking is either based on the beam power or the number of interactions.

\section{Transmission and Reflection through a Dichroic Surface in 2D}

\subsection{Configuration}

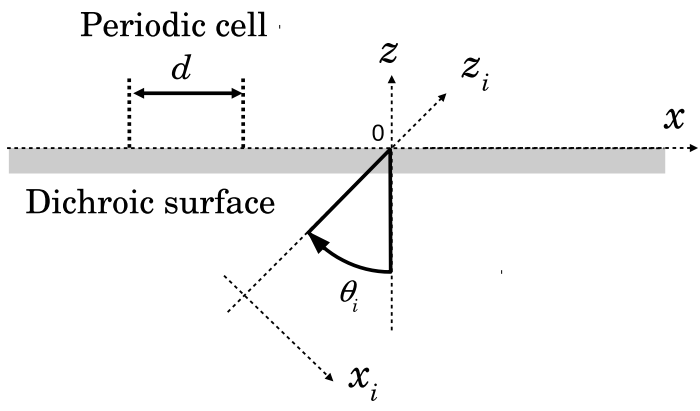

Figure 1: Configuration

In this Section, we explain how to integrate a dichroic surface in a Gaussianbeam shooting algorithm in a $2 \mathrm{D}$ case. Note that the $3 \mathrm{D}$ case could be treated in a similar way. The configuration is illustrated in Fig. 1. In a coordinate system $(x, y, z)$, the direction $y$ is chosen as the direction of invariance and the dichroic surface is placed at $z=0$. A second coordinate system $\left(x_{\mathrm{i}}, y_{\mathrm{i}}, z_{\mathrm{i}}\right)$ is associated with the incident field. Both coordinate systems are related by a rotation of angle $\theta_{\mathrm{i}}$. The polarization is so that the electric field is oriented along $y$.

The dichroic surface is assumed to be transversely periodic, of period $d \leq \lambda / 2$, where $\lambda$ is the wavelength, so that only the 0 -th order Bloch-Floquet mode is propagative. Thus, for a plane wave illumination of wavevector $\left(k_{x \mathrm{i}}, k_{z \mathrm{i}}\right)$ in the incident frame, the transmission and reflection can simply be expressed by means 
of the coefficients $T\left(k_{x \mathrm{i}}\right)$ and $R\left(k_{x \mathrm{i}}\right)$. In this article, theses coefficients are assumed to be already known. For a multilayer slab or for a grid of metal strips [8], they could be analytically calculated. In other cases, they could be computed using the finite element method, or the periodic method of moments.

\subsection{D Gaussian Beams}

The following formulation of Gaussian beams is used here. In the plane $z_{\mathrm{i}}=0$, the electric field, oriented along $y$, is given by

$$
u_{\mathrm{i}}\left(x_{\mathrm{i}}, 0\right)=a_{\mathrm{i} 0} e^{j \frac{k}{2 q_{\mathrm{i} 0}}\left(x_{\mathrm{i}}-x_{\mathrm{i} 0}\right)^{2}} e^{-j \beta_{\mathrm{i}}\left(x_{\mathrm{i}}-x_{\mathrm{i} 0}\right)} .
$$

The beam is then characterized by four parameters:

- a complex amplitude $a_{\mathrm{i} 0}$;

- a spatial shift $x_{\mathrm{i} 0}$;

- a complex curvature radius $q_{\mathrm{i} 0}$, which imaginary part can be written $k W_{\mathrm{i} 0}^{2} / 2$ where $W_{\mathrm{i} 0}$ is the beam waist size;

- a phase shift $\beta_{\mathrm{i}}$, which characterizes a beam angular tilt of $\arcsin \left(\beta_{\mathrm{i}} / k\right)$ for $\beta_{\mathrm{i}} \leq k$

From (1), two asymptotic analytical expressions can be derived. They depend either on a far-field or a paraxial approximations.

\subsection{General case}

As for the shooting algorithm, we assume that an incident Gaussian beam yields one transmitted and one reflected Gaussian beams. The transmitted and reflected beam parameters are obtained by means of a matching in the spectral domain. For the sake of conciseness, only the transmission case is presented. The transmitted field spectrum can be expressed as

$$
\tilde{u}_{\mathrm{t}}=T\left(k_{x \mathrm{i}}\right) \tilde{u}_{\mathrm{i}},
$$

where $\tilde{u}_{\mathrm{i}}$ is the Fourier transform of (1). Next, we perform the most general approximation of $T$ near $k_{x \mathrm{i}}=\beta_{\mathrm{i}}$ from which the transmitted field can be cast as a Gaussian beam. This approximation is explicitly given by

$$
T\left(k_{x \mathrm{i}}\right)=e^{T_{0}+T_{1}\left(k_{x \mathrm{i}}-\beta_{\mathrm{i}}\right)+T_{2}\left(k_{x \mathrm{i}}-\beta_{\mathrm{i}}\right)^{2}}
$$

where the coefficients $T_{0}, T_{1}$ and $T_{2}$ can be obtained via a point matching technique on three points. From this approximation, the transmitted beam parameters, i.e. $a_{\mathrm{t} 0}, x_{\mathrm{t} 0}, q_{\mathrm{t} 0}$ and $\beta_{\mathrm{i}}$, can be derived. This model is denoted GBM for Gaussian Beam Matching.

\subsection{Simplified case}

When the transmission coefficient is slowly varying with respect to $k_{x \mathrm{i}}$, the transmitted spectrum can be written as

$$
\tilde{u}_{\mathrm{t}}=T\left(\beta_{\mathrm{i}}\right) \tilde{u}_{\mathrm{i}} .
$$


Thus the transmitted beam can be approximated to the incident beam up to a multiplicative constant. This simplified model is denoted as SBM for Simplified Beam Matching.

\subsection{Special case}

When the transmission coefficient has a zero near the beam angle of incidence, the transmitted field cannot be modeled with only one Gaussian beam. We propose here a solution to treat this situation. The transmitted spectrum is firstly expressed as

$$
\tilde{u}_{\mathrm{t}}=\tilde{u}_{\mathrm{i}}+\left(T\left(k_{x \mathrm{i}}\right)-1\right) \tilde{u}_{\mathrm{i}} .
$$

Because the term $T\left(k_{x i}\right)-1$ is not vanishing, the transmitted field can efficiently be modeled by means of a sum of 2 Gaussian beams: the incident beam associated with $\tilde{u}_{\mathrm{i}}$, plus another beam associated with $\left(T\left(k_{x \mathrm{i}}\right)-1\right) \tilde{u}_{\mathrm{i}}$. The latter requires an approximation of $T\left(k_{x \mathrm{i}}\right)-1$ similar to (4). This method, denoted GBM2, can be applied in a similar way on the reflected field.

\section{Numerical experiments}

\subsection{Test-case}

We test our method on a dielectric slab of relative permittivity $\varepsilon_{\mathrm{r}}=2.1$ and thickness $h=1.13 \lambda_{\mathrm{s}}$, where $\lambda_{\mathrm{s}}$ is the wavelength in the slab. The incident Gaussian beam is such that $a_{\mathrm{i} 0}=1 \mathrm{~V} / \mathrm{m}, x_{\mathrm{i} 0}=0, q_{\mathrm{i} 0}=4 j \pi \lambda$ and $\beta_{\mathrm{i}}=0$ at a frequency of $200 \mathrm{GHz}$. Besides, the incident reference frame is rotated of an angle $\theta_{\mathrm{i}}=45^{\circ}$. Using analytical formulations, one can easily demonstrate that the reflection coefficient of the slab equals zero at an incidence of approximatively $42.44^{\circ}$. Therefore, we are in a typical situation where GBM2 is of interest.

In Fig. 2 and 3, we show the transmitted and reflected far fields computed with GBM2, SBM and the reference solution given by [9]. For the transmitted field, all the methods match. Nevertheless, the thickness of the slab implies a lateral shift of the transmitted field of $x_{\mathrm{t} 0}=0.4 \lambda$, that SBM cannot model. This notably implies an error in the far-field phase with SBM. For the reflected field, even if the field is weak, SBM yields significant errors because it does not account for the zero in the slab reflection coefficient. Conversely, GBM2 remains accurate. This shows the efficiency of replacing (4) by (5) in such situations.

\subsection{Influence of the slab thickness}

In Fig. 4, for various slab thicknesses, we display the root-mean-square error of GBM, GBM2, and SBM with respect to the reference method in the far-field. With SBM, the error rapidly increases with the slab thickness. With GBM, the error is weak but there are peaks near thicknesses for which the reflection coefficient presents a zero. With GBM2, the results are accurate regardless of the slab thickness. 


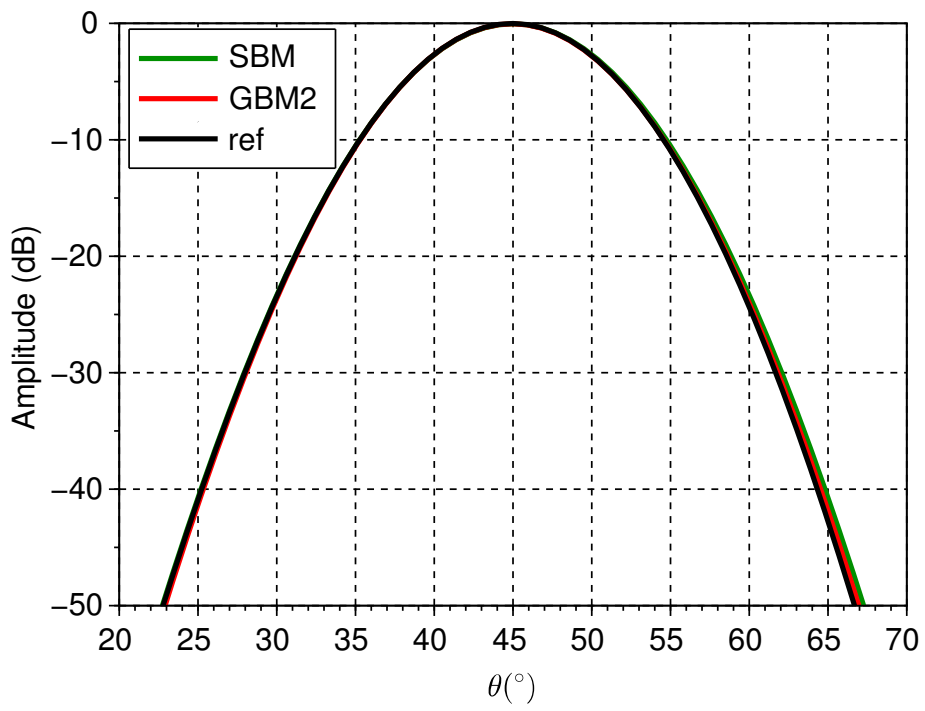

Figure 2: Normalized transmitted electric field $(\mathrm{dB})$ in the far-field zone

\section{Conclusion}

In this article, we have proposed a Gaussian-beam based method for modeling quasi-optical systems constituted by mirrors, lenses, dichroic surfaces, and horn antennas. A solution has been proposed to deal with the particular case where a dichroic surface response presents a zero near the angle of incidence. Numerical experiments have shown the efficiency of this method on a dielectric slab of various thicknesses. The final presentation should include a comparison of the complete Gaussian beam method with measurements.

\section{Acknowledgment}

The authors thank CNES and ENAC for funding this work.

\section{References}

[1] P. F. Goldsmith, I. of Electrical, E. Engineers, M. Theory, and T. Society, Quasioptical systems: Gaussian beam quasioptical propagation and applications. IEEE press New York, 1998.

[2] A. Chabory, J. Sokoloff, S. Bolioli, and K. Elis, "Application of gaussian beam based techniques to the quasi-optical systems of radiofrequency radiometers," in Eucap 2010, 2010.

[3] K. Elis, A. Chabory, J. Sokoloff, and S. Bolioli, "2D interaction of a gaussian beam with a dichroic surface for the modeling of quasi-optical systems," 


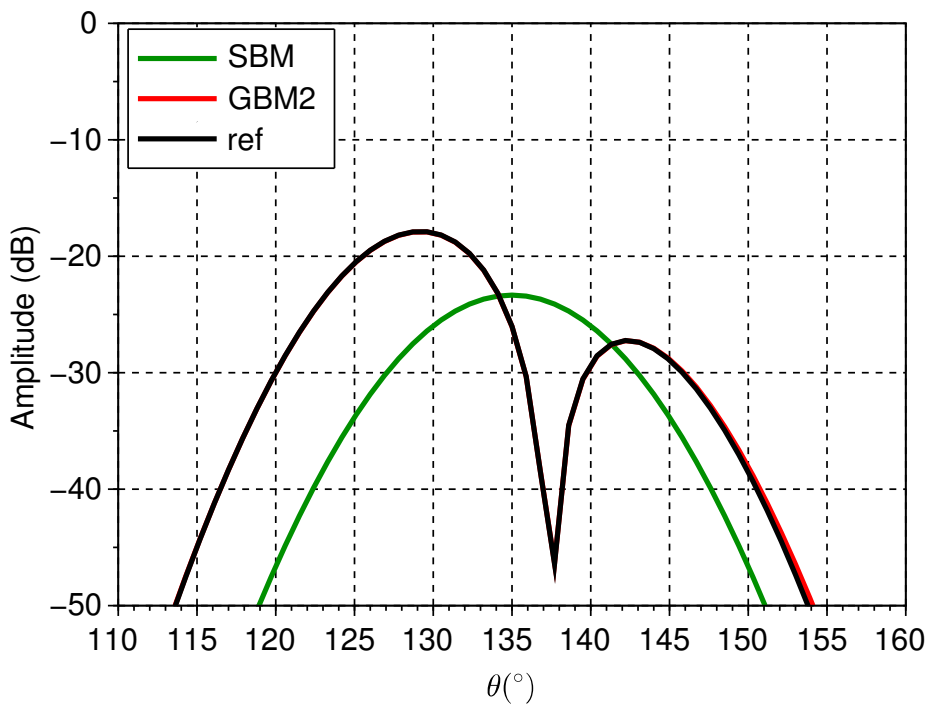

Figure 3: Normalized reflected electric field (dB) in the far-field zone

in Electromagnetics in Advanced Applications (ICEAA), 2011 International Conference on. IEEE, 2011, pp. 682-685.

[4] K. Elis, A. Chabory, and J. Sokoloff, "3D interaction of gaussian beams with dichroic surfaces for the modeling of quasi-optical systems," in Antenna Technology and Applied Electromagnetics (ANTEM), 2012 15th International Symposium on. IEEE, 2012, pp. 1-5.

[5] D. Lugara, C. Letrou, A. Shlivinski, E. Heyman, and A. Boag, "Frame-based gaussian beam summation method: Theory and applications," Radio Science, vol. 38 , no. $2,2003$.

[6] A. Chabory, J. Sokoloff, S. Bolioli, and P. F. Combes, "Computation of electromagnetic scattering by multilayer dielectric objects using gaussian beam based techniques," Comptes Rendus Physique, vol. 6, no. 6, pp. 654-662, 2005 .

[7] G. A. Deschamps, "Ray techniques in electromagnetics," Proceedings of the IEEE, vol. 60, no. 9, pp. 1022-1035, 1972.

[8] S. Tretyakov, Analytical modeling in applied electromagnetics. Artech House, 2003.

[9] J. Chen, "Computation of reflected and transmitted horn radiation patterns for a dichroic plate," TDA Progress, pp. pp 236-254, 1994. 


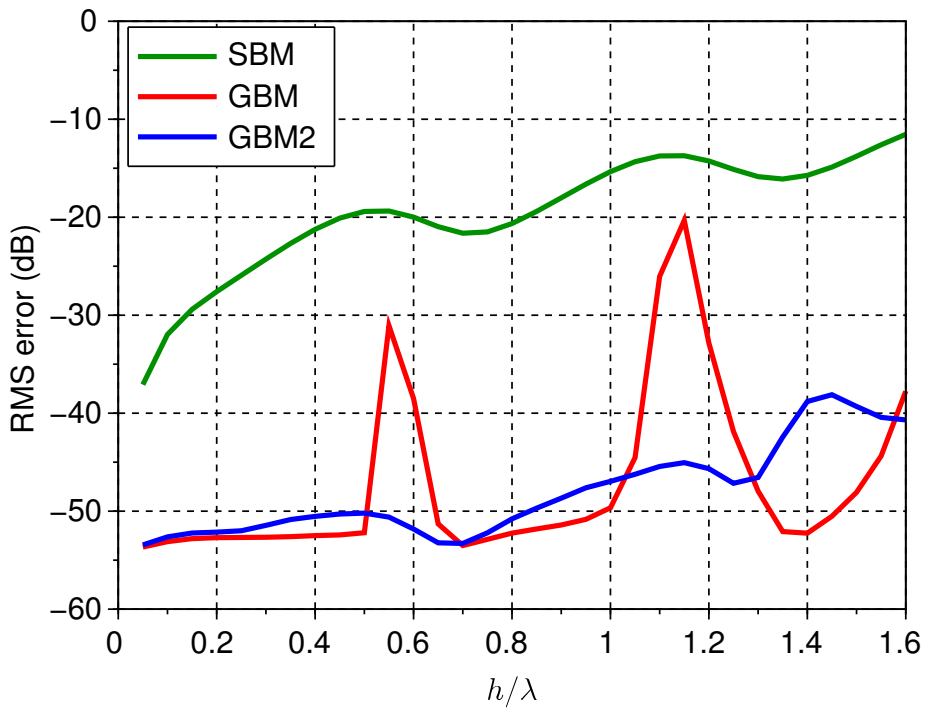

Figure 4: Root-mean-square error in the far-field zone (dB) 